\title{
On a product-type system of difference equations of second order solvable in closed form
}

\author{
Stevo Stević ${ }^{1,2^{*}}$, Bratislav Iričanin ${ }^{3}$ and Zdeněk Šmarda ${ }^{4,5}$
}

"Correspondence: sstevic@ptt.rs ${ }^{1}$ Mathematical Institute of the Serbian Academy of Sciences, Knez Mihailova 36/III, Beograd, 11000, Serbia

${ }^{2}$ Operator Theory and Applications Research Group, Department of Mathematics, King Abdulaziz University, P.O. Box 80203, Jeddah, 21589, Saudi Arabia Full list of author information is available at the end of the article

\author{
Abstract \\ It is shown that the following system of difference equations

$$
z_{n+1}=\frac{z_{n}^{a}}{w_{n-1}^{b}}, \quad w_{n+1}=\frac{w_{n}^{c}}{z_{n-1}^{d}}, \quad n \in \mathbb{N}_{0}
$$ \\ where $a, b, c, d \in \mathbb{Z}, z_{-1}, z_{0}, w_{-1}, w_{0} \in \mathbb{C}$, is solvable in closed form. \\ MSC: Primary 39A10; 39A20 \\ Keywords: system of difference equations; second order system; product-type \\ system; solvable in closed form
}

\section{Introduction}

Recently there has been a great interest in studying nonlinear difference equations and systems not stemming from differential ones (see, e.g., [1-30]). The old area of solving difference equations and systems has re-attracted recent attention (see, e.g., [1-6, 12, 15, 19-26, 28-30]). Recent Stević's idea of transforming complicated equations and systems into simpler solvable ones, used for the first time in explaining the solvability of the equation appearing in [6] (an extension of the original result can be found in [20], see also [22]), was employed in several other papers (see, e.g., [1, 2, 4, 12, 15, 19, 21, 24-26, 29, 30] and the related references therein). Another area of some recent interest, essentially initiated by Papaschinopoulos and Schinas, is studying symmetric and close to symmetric systems of difference equations (see, e.g., [5, 8-10, 13, 14, 19, 21, 23, 25, 27-29]).

Stević also essentially triggered a systematic study of non-rational concrete difference equations and systems, from one side those obtained by using the translation operator (see, e.g., [16] and also [11]) and from the other side those obtained by using max-type operators (see, e.g., $[17,18,27]$ ), see also the related references cited therein. We would like to point out that for the equations and systems in [16-18,27] only long-term behavior of their positive solutions are studied. For instance, the boundedness of positive solutions to the system

$$
x_{n+1}=\max \left\{a, \frac{y_{n}^{p}}{x_{n-1}^{q}}\right\}, \quad y_{n+1}=\max \left\{a, \frac{x_{n}^{p}}{y_{n-1}^{q}}\right\}, \quad n \in \mathbb{N}_{0},
$$

(C) 2015 Stević et al. This article is distributed under the terms of the Creative Commons Attribution 4.0 International License (http://creativecommons.org/licenses/by/4.0/), which permits unrestricted use, distribution, and reproduction in any medium, provided you give appropriate credit to the original author(s) and the source, provide a link to the Creative Commons license, and indicate if changes were made. 
with $\min \{a, p, q\}>0$, was investigated in [27]. System (1) is obviously obtained from the next product-type one

$$
x_{n+1}=\frac{y_{n}^{p}}{x_{n-1}^{q}}, \quad y_{n+1}=\frac{x_{n}^{p}}{y_{n-1}^{q}}, \quad n \in \mathbb{N}_{0} \text {, }
$$

by acting with the max-type operator $m_{a}(t)=\max \{a, t\}$ onto the right-hand sides of both equations in (2) (see also [17] and [18] for related scalar equations). Note that for the case of positive initial values, system (2) can be solved by taking the logarithm to the both sides of both equations therein, since this transforms the system to a linear second order system of difference equations with constant coefficients, which is solvable. Note that the method does not work if initial values are not positive. Let us also mention here that positive solutions to difference equations and systems are often studied since many real-life models produce such solutions (see, e.g., [7, 15, 31]). It is also interesting to note that there are max-type systems of difference equations which are solvable (see [23]). Finally, we want to note that the long-term behavior of solutions to product-type systems and those obtained from them by acting with some 'reasonable good' transformations are frequently closely related, which is another reason for studying these systems.

Hence, a natural problem is to investigate the solvability of product-type difference equations and systems with real and/or complex initial values. In [26], Stević and his collaborators started studying the problem with an approach different from the ones in [5, $21,24,25]$, but which can be regarded as a modification of some of the methods in [16-18, 27]. They showed therein that the system

$$
z_{n+1}=\frac{w_{n}^{a}}{z_{n-1}^{b}}, \quad w_{n+1}=\frac{z_{n}^{c}}{w_{n-1}^{d}}, \quad n \in \mathbb{N}_{0}
$$

where $a, b, c, d \in \mathbb{Z}$ and $z_{-1}, z_{0}, w_{-1}, w_{0} \in \mathbb{C}$, is solvable in closed form and presented numerous applications of obtained formulas.

In this paper we continue our investigation by studying the solvability of the following system of difference equations:

$$
z_{n+1}=\frac{z_{n}^{a}}{w_{n-1}^{b}}, \quad w_{n+1}=\frac{w_{n}^{c}}{z_{n-1}^{d}}, \quad n \in \mathbb{N}_{0}
$$

where $a, b, c, d \in \mathbb{Z}$ and $z_{-1}, z_{0}, w_{-1}, w_{0} \in \mathbb{C}$.

Let us mention here that although systems (3) and (4) are similar in appearance, the methods used in dealing with them are quite different.

It is easy to see that the domain of undefinable solutions [24] to system (4) is the set

$$
\mathcal{U}=\left\{\left(z_{-1}, z_{0}, w_{-1}, w_{0}\right) \in \mathbb{C}^{4}: z_{-1}=0 \text { or } z_{0}=0 \text { or } w_{-1}=0 \text { or } w_{0}=0\right\}
$$

Hence, from now on we will assume that our initial values belong to the set $\mathbb{C}^{4} \backslash \mathcal{U}$.

A solution $\left(z_{n}, w_{n}\right)_{n \geq-1}$ of system (4) is called periodic (or eventually periodic) with period $p \in \mathbb{N}$ if there is $n_{0} \geq-1$ such that

$$
\left(z_{n+p}, w_{n+p}\right)=\left(z_{n}, w_{n}\right) \quad \text { for } n \geq n_{0} .
$$


Period $p$ is prime if there is no $\hat{p} \in \mathbb{N}, \hat{p}<p$ which is a period of the solution. For $p=1$, the solution is called eventually constant (see, e.g., [32]). For some results on the topic, see, e.g., $[7,15]$ and the related references therein. If it is said that a solution of system (4) is periodic with period $p$, it will need not mean that it is prime.

A system of difference equations of the form

$$
\begin{aligned}
& z_{n}=f\left(z_{n-1}, \ldots, z_{n-k}, w_{n-1}, \ldots, w_{n-k}\right) \\
& w_{n}=g\left(z_{n-1}, \ldots, z_{n-k}, w_{n-1}, \ldots, w_{n-k}\right), \quad n \in \mathbb{N}_{0},
\end{aligned}
$$

where $k \in \mathbb{N}$, is said to be solvable in closed form if its general solution can be found in terms of initial values $z_{-i}, w_{-i}, i=\overline{1, k}$, delay $k$ and index $n$ only.

\section{Main result}

The main result in this paper is proved in this section.

Theorem 1 Assume that $a, b, c, d \in \mathbb{Z}$ and $z_{-1}, z_{0}, w_{-1}, w_{0} \in \mathbb{C} \backslash\{0\}$. Then system (4) is solvable in closed form.

Proof Case $b=0$. In this case system (4) becomes

$$
z_{n+1}=z_{n}^{a}, \quad w_{n+1}=\frac{w_{n}^{c}}{z_{n-1}^{d}}, \quad n \in \mathbb{N}_{0} .
$$

From the first equation in (5) we easily obtain

$$
z_{n}=z_{0}^{a^{n}}, \quad n \in \mathbb{N}_{0} .
$$

Employing (6) into the second equation in (5), we get

$$
w_{n}=\frac{w_{n-1}^{c}}{z_{0}^{d a^{n-2}}}
$$

for $n \geq 2$.

Hence, by using (7), we have that

$$
\begin{aligned}
w_{n} & =\frac{1}{z_{0}^{d a^{n-2}}}\left(\frac{w_{n-2}^{c}}{z_{0}^{d a^{n-3}}}\right)^{c}=\frac{w_{n-2}^{c^{2}}}{z_{0}^{d a^{n-2}+d c a^{n-3}}} \\
& =\frac{1}{z_{0}^{d a^{n-2}+d c a^{n-3}}}\left(\frac{w_{n-3}^{c}}{z_{0}^{d a^{n-4}}}\right)^{c^{2}}=\frac{w_{n-3}^{c^{3}}}{z_{0}^{d a^{n-2}+d c a^{n-3}+d c^{2} a^{n-4}}}
\end{aligned}
$$

for $n \geq 4$.

Assume that we have proved

$$
w_{n}=\frac{w_{n-k}^{c^{k}}}{z_{0}^{d a^{n-2}+d c a^{n-3}+d c^{2} a^{n-4}+\cdots+d c^{k-1} a^{n-k-1}}}
$$

for $n \geq k+1$. 
Then, by using (7) with $n \rightarrow n-k$ into (8), we get

$$
\begin{aligned}
& w_{n}=\frac{1}{z_{0}^{d a^{n-2}+d c a^{n-3}+d c^{2} a^{n-4}+\cdots+d c^{k-1} a^{n-k-1}}}\left(\frac{w_{n-k-1}^{c}}{z_{0}^{d a^{n-k-2}}}\right)^{c^{k}} \\
& =\frac{w_{n-k-1}^{c^{k+1}}}{z_{0}^{d a^{n-2}+d c a^{n-3}+d c^{2} a^{n-4}+\cdots+d c^{k-1} a^{n-k-1}+d c^{k} a^{n-k-2}}}
\end{aligned}
$$

for $n \geq k+2$.

From (7), (9) and the method of induction we see that (8) holds for every $k$ such that $1 \leq k \leq n-1$.

By taking $k=n-1$ into (8) we get

$$
w_{n}=\frac{w_{1}^{c^{n-1}}}{z_{0}^{d a^{n-2}+d c a^{n-3}+d c^{2} a^{n-4}+\cdots+d c^{n-2}}}
$$

for $n \geq 2$.

Now we have two subcases to consider.

Subcase $a \neq c$. In this case from (10) we get

$$
w_{n}=\frac{w_{1}^{c^{n-1}}}{z_{0}^{d^{\frac{a^{n-1}-c^{n-1}}{a-c}}}}, \quad n \geq 2
$$

Using the next relation

$$
w_{1}=\frac{w_{0}^{c}}{z_{-1}^{d}}
$$

in (11) we get

$$
w_{n}=\frac{w_{0}^{c^{n}}}{z_{0}^{\frac{a^{n-1}-c^{n-1}}{a-c}} z_{-1}^{d c^{n-1}}}, \quad n \in \mathbb{N} .
$$

Subcase $a=c$. In this case from (10) we get

$$
w_{n}=\frac{w_{1}^{a^{n-1}}}{z_{0}^{d(n-1) a^{n-2}}}
$$

for $n \geq 2$.

Using (12) with $a=c$ into (14), we get

$$
w_{n}=\frac{w_{0}^{a^{n}}}{z_{0}^{d(n-1) a^{n-2}} z_{-1}^{d a^{n-1}}}, \quad n \in \mathbb{N} .
$$

Case $d=0$. In this case system (4) becomes

$$
z_{n+1}=\frac{z_{n}^{a}}{w_{n-1}^{b}}, \quad w_{n+1}=w_{n}^{c}, \quad n \in \mathbb{N}_{0} .
$$


From the second equation in (16) we have that

$$
w_{n}=w_{0}^{c^{n}}, \quad n \in \mathbb{N}_{0}
$$

Employing (17) into the first equation in (16), we get

$$
z_{n}=\frac{z_{n-1}^{a}}{w_{0}^{b c^{n-2}}}
$$

for $n \geq 2$.

Hence, by using (18), we have

$$
\begin{aligned}
z_{n} & =\frac{1}{w_{0}^{b c^{n-2}}}\left(\frac{z_{n-2}^{a}}{w_{0}^{b c^{n-3}}}\right)^{a}=\frac{z_{n-2}^{a^{2}}}{w_{0}^{b c^{n-2}+b a c^{n-3}}} \\
& =\frac{1}{w_{0}^{b c^{n-2}+b a c^{n-3}}}\left(\frac{z_{n-3}^{a}}{w_{0}^{b c^{n-4}}}\right)^{a^{2}}=\frac{z_{n-3}^{a^{3}}}{w_{0}^{b c^{n-2}+b a c^{n-3}+b a^{2} c^{n-4}}}
\end{aligned}
$$

for $n \geq 4$.

Assume that we have proved

$$
z_{n}=\frac{z_{n-k}^{a^{k}}}{w_{0}^{b c^{n-2}+b a c^{n-3}+b a^{2} c^{n-4}+\cdots+b a^{k-1} c^{n-k-1}}}
$$

for $n \geq k+1$.

Then, by using (18) with $n \rightarrow n-k$ into (19), we get

$$
\begin{aligned}
z_{n} & =\frac{1}{w_{0}^{b c^{n-2}+b a c^{n-3}+b a^{2} c^{n-4}+\cdots+b a^{k-1} c^{n-k-1}}}\left(\frac{z_{n-k-1}^{a}}{w_{0}^{b c^{n-k-2}}}\right)^{a^{k}} \\
& =\frac{z_{n-k-1}^{a^{k+1}}}{w_{0}^{b c^{n-2}+b a c^{n-3}+b a^{2} c^{n-4}+\cdots+b a^{k-1} c^{n-k-1}+b a^{k} c^{n-k-2}}}
\end{aligned}
$$

for $n \geq k+2$.

From (18), (20) and the method of induction we see that (19) holds for every $k$ such that $1 \leq k \leq n-1$.

By taking $k=n-1$ into (19) we get

$$
z_{n}=\frac{z_{1}^{a^{n-1}}}{w_{0}^{b c^{n-2}+b a c^{n-3}+b a^{2} c^{n-4}+\cdots+b c a^{n-3}+b a^{n-2}}}
$$

for $n \geq 2$.

Now we have two subcases to consider.

Subcase $a \neq c$. In this case from (21) we get

$$
z_{n}=\frac{z_{1}^{a^{n-1}}}{w_{0}^{b \frac{a^{n-1}-c^{n-1}}{a-c}}}, \quad n \geq 2
$$


Using the next relation

$$
z_{1}=\frac{z_{0}^{a}}{w_{-1}^{b}}
$$

in $(22)$ we get

$$
z_{n}=\frac{z_{0}^{a^{n}}}{w_{0}^{b^{\frac{a^{n-1}-c^{n-1}}{a-c}}} w_{-1}^{b a^{n-1}}}, \quad n \in \mathbb{N} .
$$

Subcase $a=c$. In this case from (21) we get

$$
z_{n}=\frac{z_{1}^{a^{n-1}}}{w_{0}^{b(n-1) a^{n-2}}}
$$

for $n \geq 2$.

Using (23) into (25) we get

$$
z_{n}=\frac{z_{0}^{a^{n}}}{w_{0}^{b(n-1) a^{n-2}} w_{-1}^{b a^{n-1}}}, \quad n \in \mathbb{N} .
$$

Case $b d \neq 0$. First note that from the first equation in (4), for every well-defined solution, we have that

$$
w_{n-1}^{b}=\frac{z_{n}^{a}}{z_{n+1}}, \quad n \in \mathbb{N}_{0},
$$

while from the second one it follows that

$$
w_{n+1}^{b}=\frac{w_{n}^{b c}}{z_{n-1}^{b d}}, \quad n \in \mathbb{N}_{0} .
$$

Using (27) into (28) we obtain

$$
\frac{z_{n+2}^{a}}{z_{n+3}}=\frac{z_{n+1}^{a c}}{z_{n+2}^{c} z_{n-1}^{b d}}, \quad n \in \mathbb{N}_{0},
$$

which can be written as

$$
z_{n+3}=z_{n+2}^{a+c} z_{n+1}^{-a c} z_{n-1}^{b d}, \quad n \in \mathbb{N}_{0}
$$

which is a fourth order product-type difference equation.

Note also that

$$
z_{1}=\frac{z_{0}^{a}}{w_{-1}^{b}} \quad \text { and } \quad z_{2}=\frac{z_{1}^{a}}{w_{0}^{b}}=\frac{z_{0}^{a^{2}}}{w_{0}^{b} w_{-1}^{a b}}
$$

Let

$$
a_{1}=a+c, \quad b_{1}=-a c, \quad c_{1}=0, \quad d_{1}=b d .
$$


Then equation (29) can be written as

$$
z_{n+3}=z_{n+2}^{a_{1}} z_{n+1}^{b_{1}} z_{n}^{c_{1}} z_{n-1}^{d_{1}}, \quad n \in \mathbb{N}_{0}
$$

From (32) with $n \rightarrow n-1$ we get

$$
z_{n+2}=z_{n+1}^{a_{1}} z_{n}^{b_{1}} z_{n-1}^{c_{1}} z_{n-2}^{d_{1}}, \quad n \in \mathbb{N} .
$$

Employing (33) into (32) we get

$$
\begin{aligned}
z_{n+3} & =\left(z_{n+1}^{a_{1}} z_{n}^{b_{1}} z_{n-1}^{c_{1}} z_{n-2}^{d_{1}}\right)^{a_{1}} z_{n+1}^{b_{1}} z_{n}^{c_{1}} z_{n-1}^{d_{1}} \\
& =z_{n+1}^{a_{1} a_{1}+b_{1}} z_{n}^{a_{1} b_{1}+c_{1}} z_{n-1}^{a_{1} c_{1}+d_{1}} z_{n-2}^{a_{1} d_{1}} \\
& =z_{n+1}^{a_{2}} z_{n}^{b_{2}} z_{n-1}^{c_{2}} z_{n-2}^{d_{2}}
\end{aligned}
$$

for $n \in \mathbb{N}$, where

$$
a_{2}:=a_{1} a_{1}+b_{1}, \quad b_{2}:=a_{1} b_{1}+c_{1}, \quad c_{2}:=a_{1} c_{1}+d_{1}, \quad d_{2}:=a_{1} d_{1} .
$$

From (32) with $n \rightarrow n-2$ we get

$$
z_{n+1}=z_{n}^{a_{1}} z_{n-1}^{b_{1}} z_{n-2}^{c_{1}} z_{n-3}^{d_{1}}
$$

for $n \geq 2$.

Employing (36) into (34) we get

$$
\begin{aligned}
z_{n+3} & =\left(z_{n}^{a_{1}} z_{n-1}^{b_{1}} z_{n-2}^{c_{1}} z_{n-3}^{d_{1}}\right)^{a_{2}} z_{n}^{b_{2}} z_{n-1}^{c_{2}} z_{n-2}^{d_{2}} \\
& =z_{n}^{a_{1} a_{2}+b_{2}} z_{n-1}^{b_{1} a_{2}+c_{2}} z_{n-2}^{c_{1} a_{2}+d_{2}} z_{n-3}^{d_{1} a_{2}} \\
& =z_{n}^{a_{3}} z_{n-1}^{b_{3}} z_{n-2}^{c_{3}} z_{n-3}^{d_{3}}
\end{aligned}
$$

for $n \geq 2$, where

$$
a_{3}:=a_{1} a_{2}+b_{2}, \quad b_{3}:=b_{1} a_{2}+c_{2}, \quad c_{3}:=c_{1} a_{2}+d_{2}, \quad d_{3}:=d_{1} a_{2} .
$$

Assume that for some $2 \leq k \leq n$, we have proved that

$$
z_{n+3}=z_{n+3-k}^{a_{k}} z_{n+2-k}^{b_{k}} z_{n+1-k}^{c_{k}} z_{n-k}^{d_{k}}
$$

for $n \geq k-1$, and that

$$
\begin{array}{ll}
a_{k}=a_{1} a_{k-1}+b_{k-1}, & b_{k}=b_{1} a_{k-1}+c_{k-1}, \\
c_{k}=c_{1} a_{k-1}+d_{k-1}, & d_{k}=d_{1} a_{k-1} .
\end{array}
$$

Then, by using the relation

$$
z_{n+3-k}=z_{n+2-k}^{a_{1}} z_{n+1-k}^{b_{1}} z_{n-k}^{c_{1}} z_{n-1-k}^{d_{1}},
$$


for $n \geq k$, into (39) we obtain

$$
\begin{aligned}
z_{n+3} & =\left(z_{n+2-k}^{a_{1}} z_{n+1-k}^{b_{1}} z_{n-k}^{c_{1}} z_{n-1-k}^{d_{1}}\right)^{a_{k}} z_{n+2-k}^{b_{k}} z_{n+1-k}^{c_{k}} z_{n-k}^{d_{k}} \\
& =z_{n+2-k}^{a_{1} a_{k}+b_{k}} z_{n+1-k}^{b_{1} a_{k}+c_{k}} z_{n-k}^{c_{1} a_{k}+d_{k}} z_{n-1-k}^{d_{1} a_{k}} \\
& =z_{n+2-k}^{a_{k+1}} z_{n+1-k}^{b_{k+1}} z_{n-k}^{c_{k+1}} z_{n-1-k}^{d_{k+1}}
\end{aligned}
$$

for $n \geq k$, where

$$
\begin{array}{ll}
a_{k+1}:=a_{1} a_{k}+b_{k}, & b_{k+1}:=b_{1} a_{k}+c_{k}, \\
c_{k+1}:=c_{1} a_{k}+d_{k}, & d_{k+1}:=d_{1} a_{k} .
\end{array}
$$

This along with (34), (35) and the method of induction shows that (39) and (40) hold for every $2 \leq k \leq n+1$.

Hence, for $k=n+1$, we have

$$
\begin{aligned}
z_{n+3} & =z_{2}^{a_{n+1}} z_{1}^{b_{n+1}} z_{0}^{c_{n+1}} z_{-1}^{d_{n+1}} \\
& =\left(\frac{z_{0}^{a^{2}}}{w_{0}^{b} w_{-1}^{a b}}\right)^{a_{n+1}}\left(\frac{z_{0}^{a}}{w_{-1}^{b}}\right)^{b_{n+1}} z_{0}^{c_{n+1}} z_{-1}^{d_{n+1}} \\
& =z_{0}^{a^{2} a_{n+1}+a b_{n+1}+c_{n+1}} z_{-1}^{d_{n+1}} w_{0}^{-b a_{n+1}} w_{-1}^{-a b a_{n+1}-b b_{n+1}}, \quad n \in \mathbb{N}_{0} .
\end{aligned}
$$

From the recurrent relations (40) we easily obtain that the sequence $\left(a_{k}\right)_{k \geq 5}$ satisfies the difference equation

$$
a_{k}=a_{1} a_{k-1}+b_{1} a_{k-2}+c_{1} a_{k-3}+d_{1} a_{k-4} .
$$

Since $b_{k-1}=a_{k}-a_{1} a_{k-1}$ and equation (44) is linear, we have that the sequence $\left(b_{k}\right)_{k \in \mathbb{N}}$ is also a solution to equation (44). From this, the linearity of equation (44) and since $c_{k-1}=$ $b_{k}-b_{1} a_{k-1}$, we have that the sequence $\left(c_{k}\right)_{k \in \mathbb{N}}$ is also a solution to equation (44). Finally, since $d_{k}=d_{1} a_{k-1}$, the linearity of equation (44) shows that $\left(d_{k}\right)_{k \in \mathbb{N}}$ is also a solution to the equation.

Now, we show that these four sequences can be prolonged for some negative indices of use. This enables easier getting formulas for solutions to system (4).

From (42) with $k=0$ we get

$$
a_{1}=a_{1} a_{0}+b_{0}, \quad b_{1}=b_{1} a_{0}+c_{0}, \quad c_{1}=c_{1} a_{0}+d_{0}, \quad d_{1}=d_{1} a_{0} .
$$

Since $b d=d_{1} \neq 0$, from the last equation in (45) we get $a_{0}=1$. Using this fact in the first three equalities in (45), we get $b_{0}=c_{0}=d_{0}=0$.

From this and by (42) with $k=-1$ we get

$$
\begin{array}{ll}
1=a_{0}=a_{1} a_{-1}+b_{-1}, & 0=b_{0}=b_{1} a_{-1}+c_{-1}, \\
0=c_{0}=c_{1} a_{-1}+d_{-1}, & 0=d_{0}=d_{1} a_{-1} .
\end{array}
$$

Since $d_{1} \neq 0$, from the last equation in (46) we get $a_{-1}=0$. Using this fact in other three equalities in (46), we get $b_{-1}=1, c_{-1}=d_{-1}=0$. 
From this and by (42) with $k=-2$ we get

$$
\begin{array}{ll}
0=a_{-1}=a_{1} a_{-2}+b_{-2}, & 1=b_{-1}=b_{1} a_{-2}+c_{-2}, \\
0=c_{-1}=c_{1} a_{-2}+d_{-2}, & 0=d_{-1}=d_{1} a_{-2} .
\end{array}
$$

Since $d_{1} \neq 0$, from the last equation in (47) we get $a_{-2}=0$. Using this fact in other three equalities in (47), we get $b_{-2}=0, c_{-2}=1$ and $d_{-2}=0$.

From this and by (42) with $k=-3$ we get

$$
\begin{array}{ll}
0=a_{-2}=a_{1} a_{-3}+b_{-3}, & 0=b_{-2}=b_{1} a_{-3}+c_{-3}, \\
1=c_{-2}=c_{1} a_{-3}+d_{-3}, & 0=d_{-2}=d_{1} a_{-3} .
\end{array}
$$

Since $d_{1} \neq 0$, from the last equation in (48) we get $a_{-3}=0$. Using this fact in other three equalities in (48), we get $b_{-3}=0, c_{-3}=0$ and $d_{-3}=1$.

Hence, sequences $\left(a_{k}\right)_{k \geq-3},\left(b_{k}\right)_{k \geq-3},\left(c_{k}\right)_{k \geq-3}$ and $\left(d_{k}\right)_{k \geq-3}$ are solutions to linear difference equation (44) satisfying the following initial conditions:

$$
\begin{array}{llll}
a_{-3}=0, & a_{-2}=0, & a_{-1}=0, & a_{0}=1 ; \\
b_{-3}=0, & b_{-2}=0, & b_{-1}=1, & b_{0}=0 ; \\
c_{-3}=0, & c_{-2}=1, & c_{-1}=0, & c_{0}=0 ; \\
d_{-3}=1, & d_{-2}=0, & d_{-1}=0, & d_{0}=0,
\end{array}
$$

respectively.

Since difference equation (44) is solvable, it follows that closed form formulas for $\left(a_{k}\right)_{k \geq-3},\left(b_{k}\right)_{k \geq-3},\left(c_{k}\right)_{k \geq-3}$ and $\left(d_{k}\right)_{k \geq-3}$ can be found. From this fact and (43) we see that equation (29) is solvable too.

From the second equation in (4), for every well-defined solution, we have that

$$
z_{n-1}^{d}=\frac{w_{n}^{c}}{w_{n+1}}, \quad n \in \mathbb{N}_{0},
$$

while from the first one it follows that

$$
z_{n+1}^{d}=\frac{z_{n}^{a d}}{w_{n-1}^{b d}}, \quad n \in \mathbb{N}_{0} .
$$

Using (50) into (51) we obtain

$$
\frac{w_{n+2}^{c}}{w_{n+3}}=\frac{w_{n+1}^{a c}}{w_{n+2}^{a} w_{n-1}^{b d}}, \quad n \in \mathbb{N}_{0},
$$

which can be written as

$$
w_{n+3}=w_{n+2}^{a+c} w_{n+1}^{-a c} w_{n-1}^{b d}, \quad n \in \mathbb{N}_{0},
$$


which is nothing but difference equation (29). However, the sequence $\left(w_{n}\right)_{n \geq-1}$ satisfies the following initial conditions:

$$
w_{1}=\frac{w_{0}^{c}}{z_{-1}^{d}} \quad \text { and } \quad w_{2}=\frac{w_{1}^{c}}{z_{0}^{d}}=\frac{w_{0}^{c^{2}}}{z_{0}^{d} z_{-1}^{c d}} .
$$

Hence, the above presented procedure can be repeated, and it can be obtained that for $1 \leq k \leq n+1$,

$$
w_{n+3}=w_{n+3-k}^{a_{k}} w_{n+2-k}^{b_{k}} w_{n+1-k}^{c_{k}} w_{n-k}^{d_{k}}, \quad n \in \mathbb{N}_{0}
$$

where $\left(a_{k}\right)_{k \in \mathbb{N}},\left(b_{k}\right)_{k \in \mathbb{N}},\left(c_{k}\right)_{k \in \mathbb{N}}$ and $\left(d_{k}\right)_{k \in \mathbb{N}}$ satisfy recurrent relations (40) with initial conditions (31).

From (54) with $k=n+1$ and by using (53) we get

$$
\begin{aligned}
w_{n+3} & =w_{2}^{a_{n+1}} w_{1}^{b_{n+1}} w_{0}^{c_{n+1}} w_{-1}^{d_{n+1}} \\
& =\left(\frac{w_{0}^{c^{2}}}{z_{0}^{d} z_{-1}^{c d}}\right)^{a_{n+1}}\left(\frac{w_{0}^{c}}{z_{-1}^{d}}\right)^{b_{n+1}} w_{0}^{c_{n+1}} w_{-1}^{d_{n+1}} \\
& =w_{0}^{c^{2} a_{n+1}+c b_{n+1}+c_{n+1}} w_{-1}^{d_{n+1}} z_{0}^{-d a_{n+1}} z_{-1}^{-c d a_{n+1}-d b_{n+1}}, \quad n \in \mathbb{N}_{0} .
\end{aligned}
$$

Also the sequences $\left(a_{k}\right)_{k \in \mathbb{N}},\left(b_{k}\right)_{k \in \mathbb{N}},\left(c_{k}\right)_{k \in \mathbb{N}}$ and $\left(d_{k}\right)_{k \in \mathbb{N}}$ satisfy the difference equation (44) with initial conditions in (49), respectively.

As above the solvability of equation (44) shows that closed form formulas for $\left(a_{k}\right)_{k \geq-3}$, $\left(b_{k}\right)_{k \geq-3},\left(c_{k}\right)_{k \geq-3}$ and $\left(d_{k}\right)_{k \geq-3}$ can be found. This fact along with (55) implies that equation (52) is solvable too. A direct calculation shows that the sequences $\left(z_{n}\right)_{n \geq-1}$ in (43) and $\left(w_{n}\right)_{n \geq-1}$ in (55) are solutions to system (4) with initial values $z_{-1}, z_{0}$, that is, $w_{-1}, w_{0}$ respectively. Hence, system (4) is also solvable in this case, finishing the proof of the theorem.

Remark 1 Note that difference equation (44) is not only theoretically but also practically solvable since the characteristic polynomial

$$
p_{4}(\lambda)=\lambda^{4}-a_{1} \lambda^{3}-b_{1} \lambda^{2}-c_{1} \lambda-d_{1}
$$

associated to the difference equation is of fourth order, which means that we can explicitly find its roots.

Remark 2 Since we are interested in those initial values $z_{-1}, z_{0}, w_{-1}, w_{0} \in \mathbb{C}$ which uniquely define solutions to system (4), to avoid multi-valued solutions to the system, we posed the condition $a, b, c, d \in \mathbb{Z}$.

From the proof of Theorem 1 we obtain the following corollary.

Corollary 1 Consider system (4) with $a, b, c, d \in \mathbb{Z}$. Assume that $z_{-1}, z_{0}, w_{-1}, w_{0} \in \mathbb{C} \backslash\{0\}$. Then the following statements are true.

(a) If $b=0$ and $a \neq c$, then the general solution to system (4) is given by (6) and (13). 
(b) If $b=0$ and $a=c$, then the general solution to system (4) is given by (6) and (15).

(c) If $d=0$ and $a \neq c$, then the general solution to system (4) is given by (17) and (24).

(d) If $d=0$ and $a=c$, then the general solution to system (4) is given by (17) and (26).

(e) If $b d \neq 0$, then the general solution to system (4) is given by (43) and (55).

Let $\lambda_{i}, i=\overline{1,4}$, be the roots of the characteristic polynomial (56) of difference equation (44). If they satisfy the condition

$$
\lambda_{i} \neq \lambda_{j} \quad \text { for } i \neq j
$$

then it is known that a general solution to equation (44) has the following form:

$$
u_{n}=\alpha_{1} \lambda_{1}^{n}+\alpha_{2} \lambda_{2}^{n}+\alpha_{3} \lambda_{3}^{n}+\alpha_{4} \lambda_{4}^{n}, \quad n \in \mathbb{N}
$$

where $\alpha_{i}, i=\overline{1,4}$, are arbitrary constants. Since for the case $d_{1} \neq 0$ the solution can be prolonged for nonpositive indices, then we may assume that formula (57) holds also for $n \geq-3$ (or $n \geq-4$ if necessary).

In order to find, in this case, a general solution to system (4) in closed form, we will need the following known lemma. We give a proof of it for the completeness and benefit of the reader.

Lemma 1 Assume that $\lambda_{j}, j=\overline{1, k}$, are pairwise different zeros of the polynomial

$$
P(z)=\alpha_{k} z^{k}+\alpha_{k-1} z^{k-1}+\cdots+\alpha_{1} z+\alpha_{0}
$$

Then

$$
\sum_{j=1}^{k} \frac{\lambda_{j}^{l}}{P^{\prime}\left(\lambda_{j}\right)}=0
$$

for $l=\overline{0, k-2}$, and

$$
\sum_{j=1}^{k} \frac{\lambda_{j}^{k-1}}{P^{\prime}\left(\lambda_{j}\right)}=\frac{1}{\alpha_{k}}
$$

Proof The functions

$$
f_{l}(z)=\frac{z^{l}}{P(z)}, \quad l \in \mathbb{N},
$$

are meromorphic on the Riemann sphere. Hence, by the residue theorem, we have that

$$
\sum_{j=1}^{k} \operatorname{Res}_{z=\lambda_{j}} f_{l}(z)+\operatorname{Res}_{z=\infty} f_{l}(z)=0
$$

for every $l \in \mathbb{N}$. 
Now note that the Laurent expansion of $f_{l}$ at zero is

$$
f_{l}(z)=\frac{z^{l}}{\alpha_{k} \prod_{j=1}^{k}\left(z-\lambda_{j}\right)}=\frac{z^{l-k}}{\alpha_{k} \prod_{j=1}^{k}\left(1-\lambda_{j} / z\right)}=\frac{1}{\alpha_{k}} z^{l-k}+\sum_{s=1}^{\infty} b_{-s} z^{l-k-s}
$$

for some complex numbers $b_{-s}, s \in \mathbb{N}$.

On the other hand, since $\lambda_{j}, j=\overline{1, k}$, are simple poles of $f_{l}$, we have that

$$
\operatorname{Res}_{z=\lambda_{j}} f_{l}(z)=\frac{\lambda_{j}^{l}}{P^{\prime}\left(\lambda_{j}\right)}, \quad j=\overline{1, k}
$$

From this and since $\operatorname{Res}_{z=\infty} f_{l}(z)$ is equal to the negative value of the coefficient at $1 / z$ in the Laurent expansion, it follows that $\operatorname{Res}_{z=\infty} f_{l}(z)=0$ when $l=\overline{0, k-2}$ and $\operatorname{Res}_{z=\infty} f_{k-1}(z)=$ $-1 / \alpha_{k}$. Using these facts in (58) the lemma follows.

If we apply Lemma 1 to polynomial $p_{4}$ in (56), and since $p_{4}(t)=\prod_{l=1}^{4}\left(t-\lambda_{j}\right)$ (note that $\alpha_{4}=1$ ), we have

$$
\sum_{j=1}^{4} \frac{\lambda_{j}^{l}}{p_{4}^{\prime}\left(\lambda_{j}\right)}=0
$$

for $l=\overline{0,2}$, and

$$
\sum_{j=1}^{4} \frac{\lambda_{j}^{3}}{p_{4}^{\prime}\left(\lambda_{j}\right)}=1
$$

From this, since from (49) we have $a_{-3}=a_{-2}=a_{-1}=0$ and $a_{0}=1$, and a general solution of (4) has the form in (57), we obtain

$$
\begin{aligned}
a_{n}= & \sum_{j=1}^{4} \frac{\lambda_{j}^{n+3}}{p_{4}^{\prime}\left(\lambda_{j}\right)} \\
= & \frac{\lambda_{1}^{n+3}}{\left(\lambda_{1}-\lambda_{2}\right)\left(\lambda_{1}-\lambda_{3}\right)\left(\lambda_{1}-\lambda_{4}\right)}+\frac{\lambda_{2}^{n+3}}{\left(\lambda_{2}-\lambda_{1}\right)\left(\lambda_{2}-\lambda_{3}\right)\left(\lambda_{2}-\lambda_{4}\right)} \\
& +\frac{\lambda_{3}^{n+3}}{\left(\lambda_{3}-\lambda_{1}\right)\left(\lambda_{3}-\lambda_{2}\right)\left(\lambda_{3}-\lambda_{4}\right)}+\frac{\lambda_{4}^{n+3}}{\left(\lambda_{4}-\lambda_{1}\right)\left(\lambda_{4}-\lambda_{2}\right)\left(\lambda_{4}-\lambda_{3}\right)}
\end{aligned}
$$

for $n \geq-3$.

On the other hand, from (40) we get

$$
\begin{aligned}
& b_{n}=a_{n+1}-a_{1} a_{n}, \\
& c_{n}=b_{n+1}-b_{1} a_{n}, \\
& d_{n}=d_{1} a_{n-1}
\end{aligned}
$$

for $n \geq-3$. 
By using (59) into (60) we get

$$
b_{n}=\sum_{j=1}^{4} \frac{\lambda_{j}-a_{1}}{p_{4}^{\prime}\left(\lambda_{j}\right)} \lambda_{j}^{n+3}
$$

for $n \geq-3$.

By using (59) and (63) into (61) we get

$$
c_{n}=\sum_{j=1}^{4} \frac{\left(\lambda_{j}-a_{1}\right) \lambda_{j}-b_{1}}{p_{4}^{\prime}\left(\lambda_{j}\right)} \lambda_{j}^{n+3}
$$

for $n \geq-3$.

By using (59) into (62) we get

$$
d_{n}=\sum_{j=1}^{4} \frac{d_{1}}{p_{4}^{\prime}\left(\lambda_{j}\right)} \lambda_{j}^{n+2}
$$

for $n \geq-3$, where we have used the fact that (59) also holds for $n=-4$ (in fact, we may assume that equality (59) holds for every $n \geq-s$, for any fixed $s \in \mathbb{N}$, since due to the assumption $d_{1} \neq 0$, any solution of equation (44) can be prolonged for any nonpositive value of index $n)$.

By using (59), (63), (64) and (65) into (43) and (55), we get formulas for general solutions to system (4) in closed form.

Formulas obtained in this section can be used in describing the long-term behavior of solutions to system (4) in many cases. We will formulate and prove here only one result, just as an example. The formulations and proofs of other results, which are similar and whose proofs use standard techniques, we leave to the reader as some exercises.

Theorem 2 Assume that $b=c=0$ and $a, d \in \mathbb{Z}$. Then the following statements hold:

(a) If $a=1$, then every solution to system (4) is eventually constant.

(b) If $a=0$, then $z_{n}=w_{n}=1, n \geq 3$.

(c) If $a=-1$, then every solution to system (4) is two-periodic.

(d) If $a>1$ and $\left|z_{0}\right|<1$, then $z_{n} \rightarrow 0$ as $n \rightarrow \infty$.

(e) If $a>1$ and $\left|z_{0}\right|>1$, then $\left|z_{n}\right| \rightarrow \infty$ as $n \rightarrow \infty$.

(f) If $a>1$ and $\left|z_{0}^{d}\right|<1$, then $\left|w_{n}\right| \rightarrow \infty$ as $n \rightarrow \infty$.

(g) If $a>1$ and $\left|z_{0}^{d}\right|>1$, then $w_{n} \rightarrow 0$ as $n \rightarrow \infty$.

(h) If $a<-1$ and $\left|z_{0}\right|<1$, then $z_{2 n} \rightarrow 0$ as $n \rightarrow \infty$ and $\left|z_{2 n+1}\right| \rightarrow \infty$ as $n \rightarrow \infty$.

(i) If $a<-1$ and $\left|z_{0}\right|>1$, then $z_{2 n+1} \rightarrow 0$ as $n \rightarrow \infty$ and $\left|z_{2 n}\right| \rightarrow \infty$ as $n \rightarrow \infty$.

(j) If $a<-1$ and $\left|z_{0}^{d}\right|>1$, then $w_{2 n} \rightarrow 0$ as $n \rightarrow \infty$ and $\left|w_{2 n+1}\right| \rightarrow \infty$ as $n \rightarrow \infty$.

(k) If $a<-1$ and $\left|z_{0}^{d}\right|<1$, then $w_{2 n+1} \rightarrow 0$ as $n \rightarrow \infty$ and $\left|w_{2 n}\right| \rightarrow \infty$ as $n \rightarrow \infty$.

Proof (a) If we replace $a=1$ and $c=0$ in (6) and (7), we obtain $z_{n}=z_{0}$ and $w_{n+2}=1 / z_{0}^{d}$, $n \in \mathbb{N}_{0}$, from which the statement follows.

(b) By replacing $a=0$ and $c=0$ in (6) and (7), we get $z_{n}=1, n \in \mathbb{N}$ and $w_{n}=1, n \geq 3$, from which the statement follows.

(c) By replacing $a=-1$ and $c=0$ in (6) and (7), we get $z_{2 n}=z_{0}, z_{2 n+1}=\frac{1}{z_{0}}, w_{2 n}=1 / z_{0}^{d}$ and $w_{2 n+1}=z_{0}^{d}, n \in \mathbb{N}$, from which the statement follows. 
(d)-(k) From (6) and (7) with $c=0$ we get

$$
z_{n}=z_{0}^{a^{n}}, \quad w_{n}=\frac{1}{z_{0}^{d a^{n-2}}}, \quad n \geq 2 .
$$

Using the formulas in (66) all these statements easily follow.

\section{Competing interests}

The authors declare that they have no competing interests.

\section{Authors' contributions}

All authors contributed equally to the writing of this paper. All authors read and approved the manuscript.

\section{Author details}

'Mathematical Institute of the Serbian Academy of Sciences, Knez Mihailova 36/III, Beograd, 11000, Serbia. ${ }^{2}$ Operator Theory and Applications Research Group, Department of Mathematics, King Abdulaziz University, P.O. Box 80203, Jeddah, 21589, Saudi Arabia. ${ }^{3}$ Faculty of Electrical Engineering, Belgrade University, Bulevar Kralja Aleksandra 73, Beograd, 11000 , Serbia. ${ }^{4}$ CEITEC - Central European Institute of Technology, Brno University of Technology, Technická 3058/10, Brno, 616 00, Czech Republic. ${ }^{5}$ Department of Mathematics, FEEC - Faculty of Electrical Engineering and Comunication, Brno University of Technology, Technická 3058/10, Brno, 616 00, Czech Republic.

\section{Acknowledgements}

The work of Stevo Stević is supported by the Serbian Ministry of Education and Science projects III 41025 and III 44006. The work of Bratislav Iričanin is supported by the Serbian Ministry of Education and Science projects III 41025 and OI 171007. The work of Zdeňek Šmarda was realized in CEITEC - Central European Institute of Technology with research infrastructure supported by the project CZ.1.05/1.1.00/02.0068 financed from European Regional Development Fund. He was also supported by the project FEKT-S-14-2200 of Brno University of Technology.

Received: 14 August 2015 Accepted: 23 September 2015 Published online: 12 October 2015

\section{References}

1. Aloqeili, M: Dynamics of a kth order rational difference equation. Appl. Math. Comput. 181, 1328-1335 (2006)

2. Aloqeili, M: Dynamics of a rational difference equation. Appl. Math. Comput. 176, 768-774 (2006)

3. Andruch-Sobilo, A, Migda, M: On the rational recursive sequence $x_{n+1}=a x_{n-1} /\left(b+c x_{n} x_{n-1}\right)$. Tatra Mt. Math. Publ. 43, 1-9 (2009)

4. Bajo, I, Liz, E: Global behaviour of a second-order nonlinear difference equation. J. Differ. Equ. Appl. 17, 1471-1486 (2011)

5. Berg, L, Stević, S: On some systems of difference equations. Appl. Math. Comput. 218, 1713-1718 (2011)

6. Cinar, C: On the positive solutions of difference equation. Appl. Math. Comput. 150(1), 21-24 (2004)

7. Grove, EA, Ladas, G: Periodicities in Nonlinear Difference Equations. Chapman \& Hall/CRC Press, Boca Raton (2005)

8. Papaschinopoulos, G, Schinas, CJ: On a system of two nonlinear difference equations. J. Math. Anal. Appl. 219(2), 415-426 (1998)

9. Papaschinopoulos, G, Schinas, CJ: On the behavior of the solutions of a system of two nonlinear difference equations. Commun. Appl. Nonlinear Anal. 5(2), 47-59 (1998)

10. Papaschinopoulos, G, Schinas, CJ: Invariants for systems of two nonlinear difference equations. Differ. Equ. Dyn. Syst. 7(2), 181-196 (1999)

11. Papaschinopoulos, $G$, Schinas, $C J$, Stefanidou, G: On the nonautonomous difference equation $x_{n+1}=A_{n}+\left(x_{n-1}^{p} / x_{n}^{q}\right)$. Appl. Math. Comput. 217, 5573-5580 (2011)

12. Papaschinopoulos, G, Stefanidou, G: Asymptotic behavior of the solutions of a class of rational difference equations. Int. J. Difference Equ. 5(2), 233-249 (2010)

13. Stefanidou, G, Papaschinopoulos, G, Schinas, C: On a system of max difference equations. Dyn. Contin. Discrete Impuls. Syst. 14(6), 885-903 (2007)

14. Stefanidou, G, Papaschinopoulos, G, Schinas, CJ: On a system of two exponential type difference equations. Commun. Appl. Nonlinear Anal. 17(2), 1-13 (2010)

15. Stević, S: A short proof of the Cushing-Henson conjecture. Discrete Dyn. Nat. Soc. 2006, Article ID 37264 (2006)

16. Stević, S: Boundedness character of a class of difference equations. Nonlinear Anal. TMA 70, 839-848 (2009)

17. Stević, S: On a generalized max-type difference equation from automatic control theory. Nonlinear Anal. TMA 72, 1841-1849 (2010)

18. Stević, S: On a nonlinear generalized max-type difference equation. J. Math. Anal. Appl. 376, 317-328 (2011)

19. Stević, S: On a system of difference equations. Appl. Math. Comput. 218, 3372-3378 (2011)

20. Stević, S: On the difference equation $x_{n}=x_{n-2} /\left(b_{n}+c_{n} x_{n-1} x_{n-2}\right)$. Appl. Math. Comput. 218, 4507-4513 (2011)

21. Stević, S: On a third-order system of difference equations. Appl. Math. Comput. 218, 7649-7654 (2012)

22. Stević, S: On the difference equation $x_{n}=x_{n-k} /\left(b+c x_{n-1} \cdots x_{n-k}\right)$. Appl. Math. Comput. 218, 6291-6296 (2012)

23. Stević, S: Solutions of a max-type system of difference equations. Appl. Math. Comput. 218, $9825-9830$ (2012)

24. Stević, S: Domains of undefinable solutions of some equations and systems of difference equations. Appl. Math. Comput. 219, 11206-11213 (2013)

25. Stević, S: Representation of solutions of bilinear difference equations in terms of generalized Fibonacci sequences. Electron. J. Qual. Theory Differ. Equ. 2014, Article No. 67 (2014) 
26. Stević, S, Alghamdi, MA, Alotaibi, A, Elsayed, EM: Solvable product-type system of difference equations of second order. Electron. J. Differ. Equ. 2015, Article No. 169 (2015)

27. Stević, S, Alghamdi, MA, Alotaibi, A, Shahzad, N: Boundedness character of a max-type system of difference equations of second order. Electron. J. Qual. Theory Differ. Equ. 2014, Article No. 45 (2014)

28. Tollu, DT, Yazlik, Y, Taskara, N: On fourteen solvable systems of difference equations. Appl. Math. Comput. 233, 310-319 (2014)

29. Stević, S, Diblik, J, Iričanin, B, Šmarda, Z: On a solvable system of rational difference equations. J. Differ. Equ. Appl. 20(5-6), 811-825 (2014)

30. Stević, S, Diblik, J, Iričanin, B, Šmarda, Z: Solvability of nonlinear difference equations of fourth order. Electron. J. Differ. Equ. 2014, Article No. 264 (2014)

31. Brauer, F, Castillo-Chavez, C: Mathematical Models in Population Biology and Epidemiology. Springer, Berlin (2012)

32. Iričanin, B, Stević, S: Eventually constant solutions of a rational difference equation. Appl. Math. Comput. 215, 854-856 (2009)

Submit your manuscript to a SpringerOpen ${ }^{\circ}$ journal and benefit from:

- Convenient online submission

Rigorous peer review

- Immediate publication on acceptance

- Open access: articles freely available online

- High visibility within the field

- Retaining the copyright to your article 